\title{
Musgos del valle seco del Patía, suroccidente de Colombia: riqueza, ecología y biogeografía
}

\author{
Mosses of the dry valley of Patía, southwestern Colombia: \\ Richness, ecology and biogeography
}

Bernardo R. Ramírez-Padilla, Verónica Solarte-Téllez y Paula A. Ramírez-Burbano

\section{Resumen}

Estudiamos los musgos que crecen en el valle del río Patía, en el suroccidente colombiano, que comprende zonas de bosque muy seco tropical y bosque seco premontano. En la franja altitudinal comprendida entre 550 y $1500 \mathrm{~m}$ s. n. m., encontramos 75 especies de musgos (28 familias y 53 géneros), 34 de ellas acrocárpicas (11 familias y 23 géneros) y 41 pleurocárpicas (17 familias y 30 géneros). Las familias con mayor número de especies son: Bryaceae (10), Pottiaceae (9), Hypnaceae (6), Macromitriaceae (5), Brachytheciaceae (5), Entodontaceae (5), Fissidentaceae (5) y Sematophyllaceae (4). Los géneros mejor representados son Bryum (6), Fissidens (5), Sematophyllum (4) y Entodon (3). El 60 \% de las especies se encuentran en un solo tipo de sustrato: suelo (31), corteza (10), materia orgánica (3) y roca (1); el 40 \% restante se encuentra en 2 o más sustratos. Bosques, matorrales, taludes y corrientes de agua son los hábitats preferidos por los musgos. Los elementos dominantes son neotropicales extraandinos (35 spp.), cosmopolitas (11), ampliamente tropicales (8) y afroamericanos (5).

Palabras clave. Briófitos. Hábitats. Sustratos. Zonas de vida.

\begin{abstract}
We studied the mosses that occur in the Patía River valley, in southwestern Colombia, which includes very dry tropical forest and premontane dry forest. In the altitudinal range between 550 and $1500 \mathrm{~m}$ a. s. 1., we found 75 species of mosses (28 families and 53 genera), 34 of them acrocarpic (11 families and 23 genera) and 41 pleurocarpic (17 families and 30 genera). The families with the greatest number of species are: Bryaceae (10), Pottiaceae (9), Hypnaceae (6), Macromitriaceae (5), Brachytheciaceae (5), Entodontaceae (5), Fissidentaceae (5) and Sematophyllaceae (4). Bryum (6), Fissidens (5), Sematophyllum (4) and Entodon (3) are the best represented genera. Sixty percent of the species are found in only one type of substrate: soil (31), bark (10), organic matter (3) and rock (1); the remaining $40 \%$ are in two or more substrates. Forest, bush, banks and water streams are the habitats preferred by the mosses. The dominant elements are extra-Andean Neotropical (35 spp.), cosmopolitan (11), widely tropical (8) and African-American (5).
\end{abstract}

Keywords. Bryophytes. Habitats. Life zones. Substrates. 


\section{Introducción}

En el Neotrópico existen 3869 especies de musgos, número que puede reducirse a medida que avancen los estudios taxonómicos y se coloquen en sinonimia muchos de los nombre conocidos en la actualidad (Delgadillo, 2000). En Colombia hay 934 especies, la mayoría de ellas presentes en la franja Andina (931 spp.) y con muy pocas en tierras bajas y cálidas; por debajo de los $500 \mathrm{~m}$ de altitud hay solamente 33 especies que pueden considerarse exclusivas de esta área (Churchill, 2009, 2015). Se ha establecido que la riqueza de especies varía a lo largo de un gradiente altitudinal de manera inversa a la que se presenta en plantas vasculares: en altitudes bajas la riqueza es menor y a medida que se asciende por las cordilleras aumenta hasta alcanzar el máximo en la franja comprendida entre 2600 y 3300 m s. n. m.; una segunda zona diversa se halla entre 2000 y 2600 m s. n. m. y una tercera en el páramo (Churchill, 1991). En la franja tropical, comprendida entre 0 y 1000 m s. n. m., sobresalen, por su riqueza en especies, las familias Pilotrichaceae, Fissidentaceae y Calymperaceae, en tanto que en la franja subandina, entre 1000 y $2350 \mathrm{~m}$ s. n. m., destacan Dicranaceae, Pilotrichaceae y Sematophyllaceae (Aguirre, 2008).

Son múltiples los factores que se han empleado para explicar la riqueza de especies de musgos de una localidad determinada. Se han considerado como importantes el $\mathrm{pH}$ del suelo, la pendiente del terreno, la incidencia lumínica y la variabilidad estructural que provee una gran variedad de ambientes y sustratos y que regula la incidencia del viento y evita la desecación (Sagar \& Wilson, 2009; Corrales et al., 2010; Tullus et al., 2012). En áreas uniformes las variaciones del microhábitat son más importantes que los elementos macroecológicos para el establecimiento de briófitos en general (Weibull, 2001; Acebey et al., 2003; Weibull \& Rydin, 2005).

Una de las características sobresalientes de los musgos es la capacidad de desecarse sin morir. Los gametofitos pueden perder casi toda el agua libre hasta alcanzar un equilibrio con la atmósfera, pero después de un periodo de rehidratación por la lluvia o el rocío reasumen el crecimiento normal (Proctor et al., 2007). Los musgos que crecen en sitios abiertos y expuestos presentan una gran tolerancia a la desecación; no obstante, aquellos que crecen sobre ramas son, por lo general, más tolerantes a la desecación rápida y frecuente y a la desecación severa (Franks \& Bergstrom, 2000). Las especies con más tolerancia corresponden a aquellas que ocupan superficies de rocas y suelo árido expuestos, con extrema sequía e incidencia directa de los rayos solares.

En su mayoría, los musgos de áreas desérticas y semidesérticas, situadas por fuera del Neotrópico, dependen de los periodos de humedad anual para cumplir sus ciclos de vida; para su crecimiento requieren por lo menos de la presencia de lluvia estacional regular. Los ciclos sexuales, desde la producción de gametofitos hasta la dehiscencia de cápsulas, son cortos y se presentan solamente durante la estación de lluvias, razón por la cual generalmente no se hallan esporofitos, mostrando una aparente ausencia de reproducción sexual (Makinde \& Odu, 1994; Pelayo, 2010; Oyesiku, 2016). Otra estrategia de supervivencia encontrada en musgos de desierto es el alargamiento de los periodos de maduración del anteridio y la dormancia del esporofito durante varios meses hasta que las condiciones de humedad apropiadas aparezcan (Stark, 2002). La carencia de información no permite establecer comparaciones con respecto al comportamiento fenológico entre los musgos de áreas secas neotropicales y los pertenecientes a otras áreas secas extraneotropicales.

En este artículo discutimos aspectos de los musgos del valle del río Patía, un enclave seco en el suroccidente de Colombia. Analizamos la riqueza de especies, su preferencia de sustratos y hábitats y los elementos biogeográficos de la flora de musgos de la zona.

\section{Materiales y métodos}

Área de estudio. El valle del Patía está ubicado entre las cordilleras Occidental y Central en el sur del departamento del Cauca y al nororiente del departamento de Nariño. Tiene una extensión de aproximadamente 120 km de largo y 20 km de ancho; está conformado por planicies bajas situadas entre 550 y $600 \mathrm{~m} \mathrm{s.} \mathrm{n.} \mathrm{m.,} \mathrm{algunas}$ colinas irregulares que pueden alcanzar los $900 \mathrm{~m} \mathrm{~s}$. $\mathrm{n}$. 
m. y dos mesetas, la de Mercaderes y la de El Bordo con elevaciones entre 1000 y 1250 m s. n. m. (Ramírez et al., 2015). Se encuentran dos zonas de vida (según el sistema de Holdridge, 2010): bosque muy seco tropical (bms-T) en las partes bajas del valle y bosque seco premontano (bs-PM) hacia las faldas de las cordilleras. La precipitación promedio anual varía localmente entre 800 y 1000 $\mathrm{mm}$ anuales, pero pueden hallarse algunos sitios con precipitación un tanto mayor (CRC, 2004). Es notoria la presencia de corrientes de agua procedentes de las partes altas de las cordilleras; entre ellas sobresalen los ríos Patía, Sajandí, Mamaconde, Guachicono, San Jorge, Mayo y Juanambú y algunas quebradas como Las Tallas,
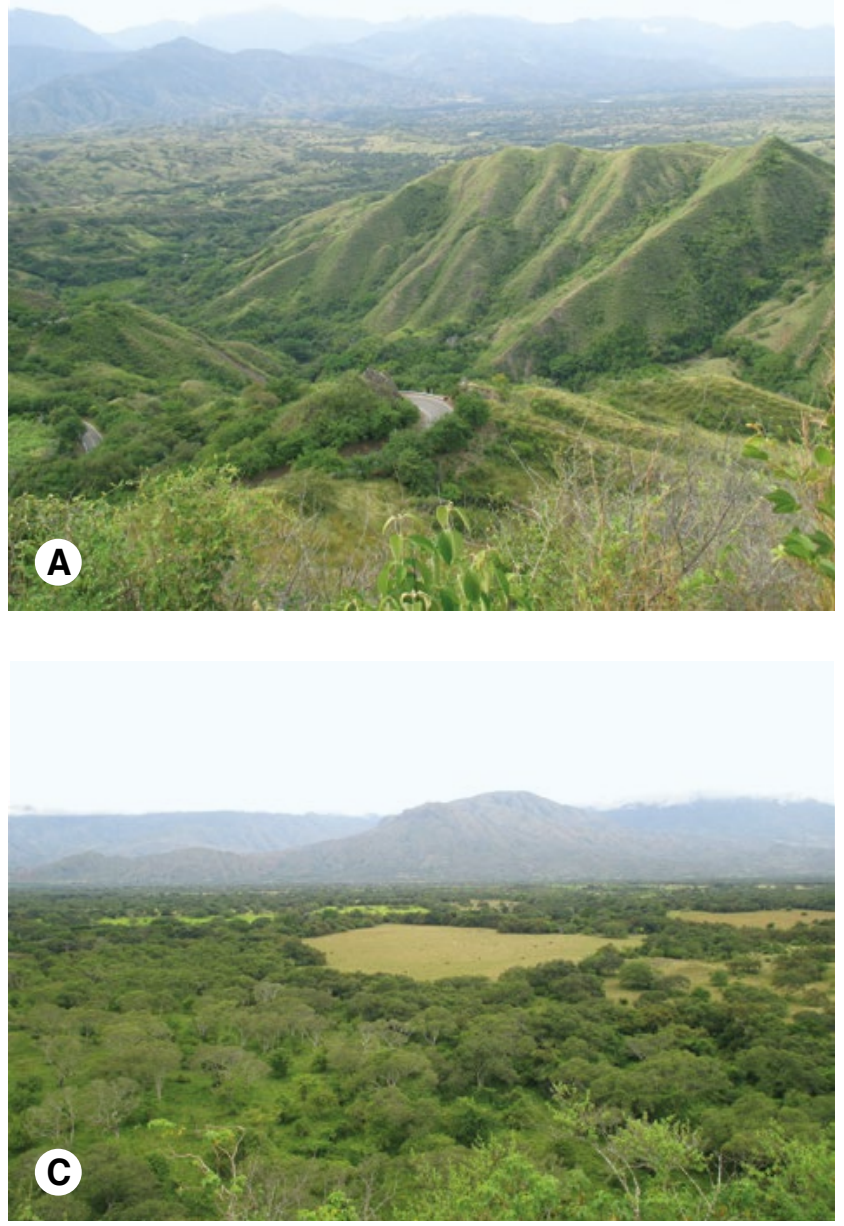

Mojarras, Cazasapos, Bonilla, La Estancia, El Silencio y Matacea (IGAC, 1992).

En las mesetas y sector norte-bajo del valle predominan pastizales y campos de cultivo que se intercalan con matorrales y remanentes boscosos, especialmente hacia las vegas de los ríos. El sector sur tiene vegetación subxerofítica con predominio de arbustos espinosos y cardonales (Figura 1). A pesar de las restricciones climáticas, el valle del Patía presenta una gran riqueza de plantas vasculares; hasta el momento se tienen registros de 1024 especies que corresponden tanto a plantas nativas como a cultivadas y naturalizadas (Ramírez et al., 2015).
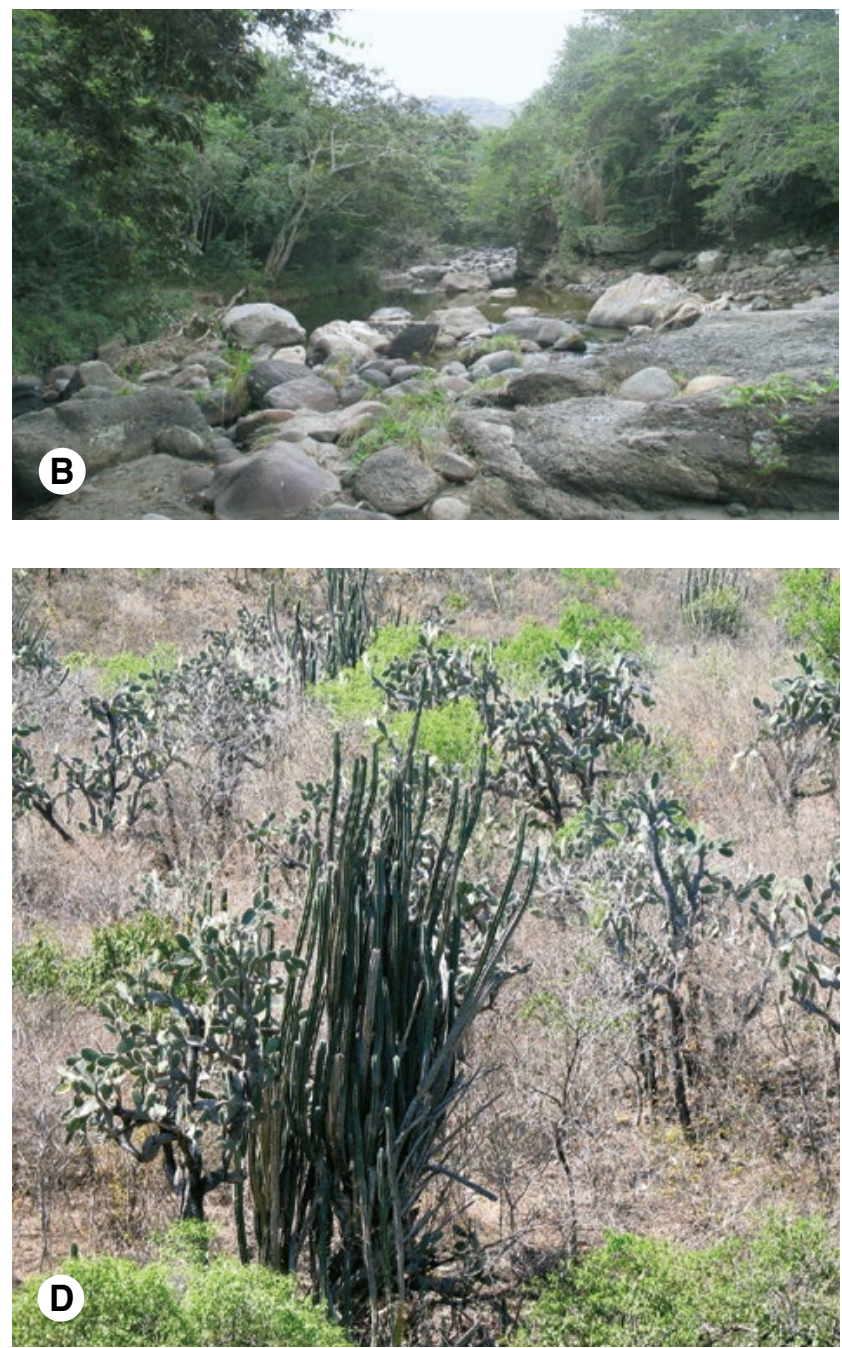

Figura 1. Ambientes del valle del río Patía, Colombia, con presencia de musgos. A) Colinas y bordes de carreteras. B) Bosques de galería y orillas de corrientes de agua. C) Áreas boscosas y potreros y D) Áreas subxerofíticas en el sur del valle. 
Trabajo de campo. Se revisaron 180 ejemplares de musgos depositados en el Herbario de la Universidad del Cauca (CAUP) y provenientes del valle del Patía, de los municipios de Bolívar, Mercaderes, Patía y Sucre pertenecientes al departamento del Cauca y de los municipios de El Rosario y Taminango pertenecientes al departamento de Nariño; los ejemplares revisados corresponden a la franja altitudinal comprendida entre 500 y 1500 m s. n. m.; además se consultaron fuentes bibliográficas y se hizo recolección libre adicional de 71 ejemplares en algunos sitios del corregimiento de Guachicono (Bolívar), corregimiento de Mojarras (Mercaderes), trayecto la Lupa-Guachicono, corregimientos de La Fonda y Piedrasentada (Patía). La identificación de las especies se basó en las anotaciones hechas por especialistas (Steven P. Churchill, Howard A. Crum, Jean Peter Frahm. Dana Griffin III, Ronald Pursell, William D. Reese, Dale Vitt y Richard H. Zander) sobre los especímenes de herbario. Otros especímenes se determinaron empleando las claves de Sharp et al. (1994) y Churchill \& Linares (1995).

Procesamiento de la información. La información procedente de cada ejemplar herborizado se organizó teniendo en cuenta los siguientes campos: especie, sustrato, hábitat en que fue encontrado y altitud del sitio de muestreo. El tratamiento taxonómico se hizo siguiendo a Churchill (2016). Para la escritura correcta de los nombres se recurrió a Tropicos Moss (www.tropicos.org). Los datos se procesaron para obtener riqueza por taxones, distribución por zonas de vida (franja tropical entre 500 y 1000 m s. n. m. y franja premontana entre 1000 y 1500 m s. n. m.), hábitats y preferencia de sustratos (suelo, rocas, corteza, materia orgánica y materiales de construcción). Se consideraron como hábitats los siguientes ambientes: corrientes de agua, áreas boscosas (bosques de galería, bosques y matorrales alejados de las corrientes de agua), taludes de caminos y carreteras, espacios abiertos y áreas ruderales. Las especies plenamente determinadas se agruparon por elementos biogeográficos siguiendo a Griffin (1990), Churchill \& Linares (1995) y Churchill (2016).

\section{Resultados y discusión}

En el valle seco del Patía se encontraron 75 especies de musgos (28 familias y 53 géneros), equivalentes al $17.5 \%$ del total conocido para el departamento del Cauca y que incrementan en 51 el número de registros en el valle (Ramírez, 2013); 34 especies corresponden a musgos acrocárpicos (11 familias y 23 géneros) y 41 a musgos pleurocárpicos (17 familias y 30 géneros). Las familias con mayor número de especies son: Bryaceae, con 10 especies (13.3\%), y Pottiaceae, con 9 (12\%), ambas constituidas por elementos acrocárpicos y que se encuentran entre las dominantes en muchas áreas xerofíticas y subxerofíticas del mundo (Clark, 2012; Dauphin \& Grayum, 2005; Downing \& Selkirk, 1993; Pelayo, 2010). Sus miembros se consideran como indicadores de ambientes perturbados y crecen en grupos pequeños, por lo general a la sombra de especies de mayor tamaño (Vilas \& Passos, 1998; Pinzón \& Linares, 2006; Pelayo, 2010; Clark, 2012). También se destacan Hypnaceae, con 6 especies (8 \%), Macromitriaceae, Brachytheciaceae, Entodontaceae y Fissidentaceae, con 5 (6.6 \% cada una), y Sematophyllaceae con 4 (5.3\% cada una). Las restantes familias se hallan representadas por 1 o 2 especies. Están constituidas por una única especie 15 familias (53.5 \%) y $3(10.7 \%)$ lo están por 2 especies. Los géneros con mayor número de especies son Bryum (6), Fissidens (5), Sematophyllum (4) y Entodon (3), que comprenden el $24 \%$ del total de especies del valle seco del Patía.

Se ha considerado que en el bosque seco la diversidad de briófitos es muy baja (Churchill, 1989; Uribe \& Gradstein, 1999; Aguirre \& Avendaño, 2008). Sin embargo, en el país son pocos los trabajos adelantados en este tipo de ecosistema que permitan confirmar la anterior aseveración. En los Montes de María (Colosó, Sucre), considerado como uno de los fragmentos de bosque seco tropical mejor conservados, se encontraron 38 especies de musgos, 22 correspondientes a elementos reofílicos (García et al., 2015, 2017). Esta zona comparte 12 especies con el valle del Patía: Philonotis uncinata, Erpodium coronatum, Fabronia ciliaris, Fissidens flaccidus, Chrysohypnum diminutivum, Meteorium nigrescens, Neckeropsis undulata, Octoblepharum albidum, Dolotortula mniifolia, Hyophila involuta, Sematophyllum subpinnatum y Thuidium tomentosum. Por otra parte, en la región subxerofítica de La Herrera, situada entre 2550 y 2900 m de altitud, con cobertura vegetal discontinua formada por hierbas, matorrales y cardonales, se encontraron 14 familias, 27 géneros y 54 especies de musgos (Pinzón \& Linares, 2001); en ambos 
casos los valores son menores a los encontrados en el valle seco del Patía, posiblemente por diferencias en la amplitud del rango altitudinal abarcado y en las condiciones medioambientales de cada sitio.

Algunas investigaciones sobre riqueza de briófitos en áreas xerofíticas y subxerofíticas de Norteamérica muestran cifras más altas que las del valle del Patía: en el Parque Nacional Big Bend, en Texas, incluido dentro del desierto Chihuahuense, se han encontrado 58 géneros y 106 especies de musgos (Magill, 1976); para los Montes Santa Mónica adyacentes al océano Pacífico, en los Ángeles, California, se tiene un registro de 122 especies (Sagar \& Wilson, 2009); en el Parque Nacional del Gran Cañón, en Arizona, se encontraron 144 especies (Clark, 2012). Los anteriores datos muestran claramente la riqueza de musgos que puede encontrarse en áreas secas y que para el caso de Colombia está en espera de establecerse con más detalle.
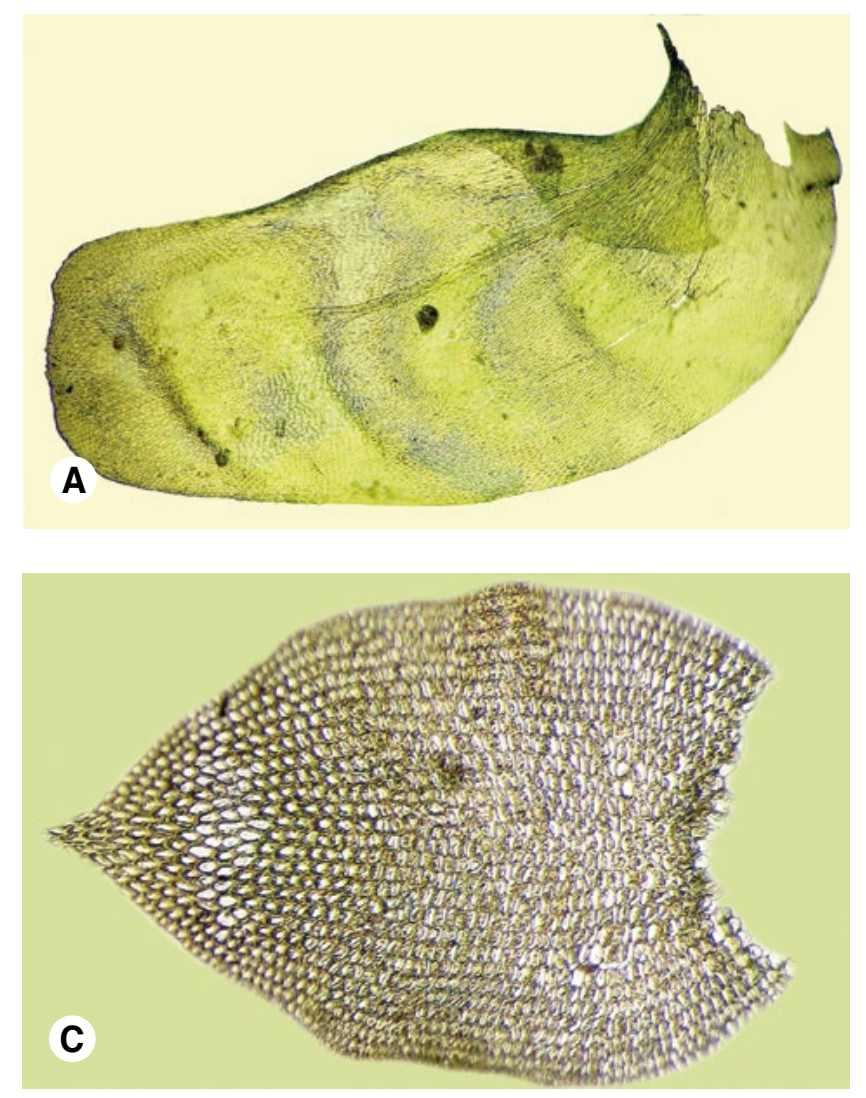

Doce especies de musgos (Brachythecium conostomum, Calymperes rubiginosum, Erpodium becarii, Lepidopilum stillicidiorum, Syringothecium sprucei, Taxiphyllum taxirameum, Macrocoma frigida, Macromitrium contextum, Dolotortula mnifolia, Trichostomum brachydontium, Entodontopsis leucostega y Stereophyllum radiculosum) corresponden a primeros registros para el departamento del Cauca, con lo cual el número total de especies para el departamento se aumenta a 440 (Ramírez, 2013). Una de estas especies, Erpodium beccarii se registra por primera vez para Colombia. Por otra parte, las especies: Entodontopsis leucostega, Orthostichopsis praetermissa, Sematophyllum tequendamae y Stereophyllum radiculosum corresponden a primeros registros para el departamento de Nariño, elevando a 396 el número de especies registradas para este departamento (Ramírez \& Churchill, 2002). En la Figura 2 se muestran algunas de las especies de musgos presentes en el valle del Patía.

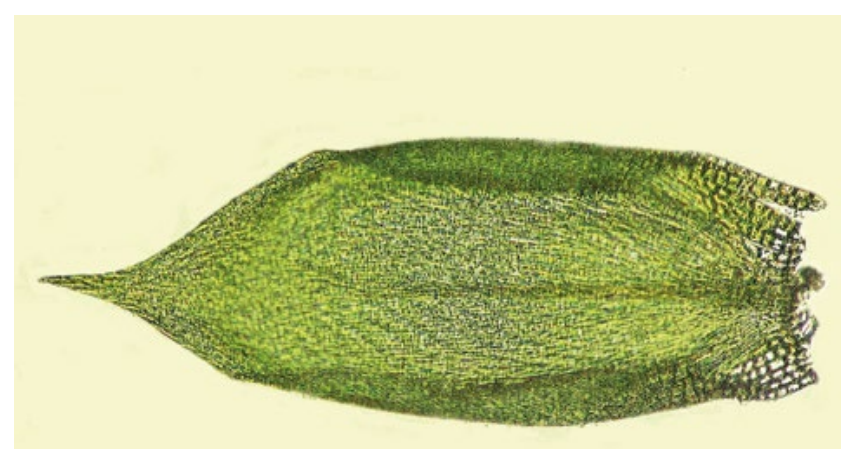

B

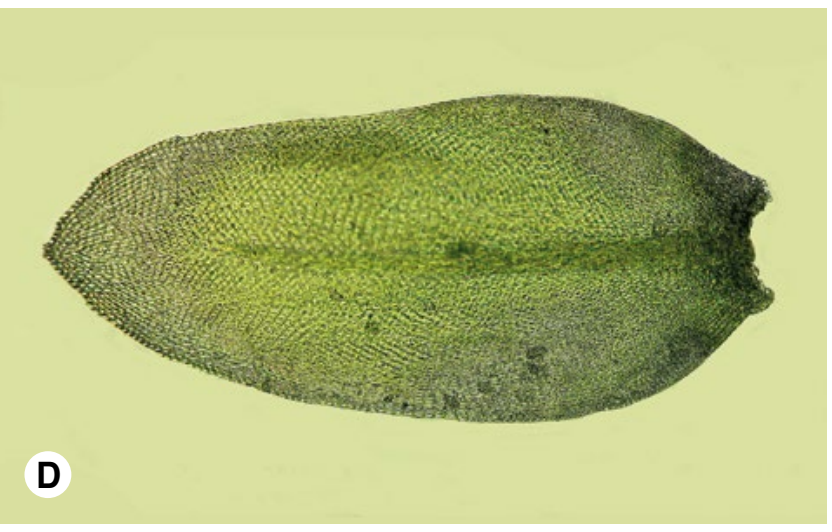

Figura 2. Hojas de algunas especies de musgos presentes en el valle del Patía, Colombia: A) Neckeropsis undulata (Neckeraceae). B) Entodontopsis leucostega (Stereophyllaceae). C) Erpodium coronatum (Erpodiaceae). D) Stereophyllum radiculosum (Stereophyllaceae). 
La franja tropical del valle presenta 46 especies de musgos; de ellas 20 corresponden a elementos encontrados localmente solo en esta franja; la franja premontana es ligeramente más rica, con 55 especies, de las cuales 29 son exclusivas de esta, en tanto que comparte 26 con la anterior. Churchill \& Linares (1995) mencionan que en Colombia, en la franja tropical, se han encontrado 163 especies, de las cuales tan solo 12 son características, y para la franja premontana se conocen 230 especies ( 13 comunes con la franja tropical) y 18 características. De igual manera anotan que en el bosque seco tropical pueden crecer sobre rocas y troncos los géneros Entodontopsis, Erpodium, Haplocladium, Helicodontium, Helicophyllum y Stereophyllum, cuatro de ellos (Entodontopsis, Erpodium, Helicophyllum y Stereophyllum) encontrados en el presente trabajo. Es de resaltar que la mayoría de las especies halladas en la franja tropical no son exclusivas de esta zona de vida como puede constatarse con los registros que se tienen de otros lugares de Colombia; únicamente Calymperes rubiginosum, Erpodium beccarii y Erpodium coronatum se pueden considerar especies restringidas a la zona de vida tropical; en la zona de vida premontana ninguna de las especies encontradas puede considerarse como exclusiva de esta franja (Churchill \& Linares, 1995; Churchill, 2016).

Considerando la preferencia de hábitats encontramos que en las corrientes de agua (taludes y rocas) existen 28 especies (12 exclusivas de este ambiente), en las áreas boscosas (bosques de galería, matorrales y bosques alejados de corrientes de agua) 34 especies (9 exclusivas), en taludes de carreteras y caminos 31 especies (14 exclusivas), en espacios abiertos 15 especies ( 3 exclusivas) y en áreas ruderales 18 especies (7 exclusivas). Bosques y matorrales, taludes y corrientes de agua son los hábitats preferidos por los musgos, y es allí donde se halla el mayor número de especies. La mayoría de ellas (45) prefieren uno solo de los 5 hábitats mencionados, 16 especies se hallan en 2 hábitats, 6 especies en 3 hábitats, 6 en 4 hábitats y una única especie, Fabronia ciliaris, puede encontrarse en cualquiera de los hábitats citados, en concordancia con lo expuesto por Ignatova et al. (2017).
En los bosques secos, el suelo, los troncos caídos y las rocas son los microhábitats más importantes para los musgos (Dauphin \& Grayum, 2005); la colonización de la corteza de arbustos y árboles es considerada como una adaptación hacia el aprovechamiento del agua sobrante de las plantas hospederas (Pócs, 1982). Al parecer, en el valle del Patía todos los hábitats ofrecen las condiciones básicas para el establecimiento de distintos taxones de musgos; la disponibilidad de diversos sustratos ofrece oportunidades de colonización a las diferentes especies. Se encontraron 45 especies con preferencia por un único tipo de sustrato; no obstante también se encontraron 30 especies colonizando 2 o más sustratos. En el sustrato suelo se hallaron 55 especies (31 de ellas restringidas a este tipo de sustrato), en corteza de árboles y arbustos 30 especies (10 restringidas), en roca 20 especies ( 1 restringida), en materia orgánica derivada de troncos, ramas y hojarasca en descomposición, 19 especies (3 restringidas) y en materiales de construcción (concreto, ladrillos) únicamente se encontraron 2 especies, ninguna característica de este tipo de sustrato (ver Anexo 1). Un total de 13 especies se encuentran en 2 sustratos, 10 en 3, 5 en 4 y únicamente una especie, Erythrodontium squarrosum aprovecha todos los sustratos disponibles.

Es conocido que en Colombia los musgos prefieren los sustratos terrestres (suelo, rocas), con especies pertenecientes a las familias Dicranaceae, Bartramiaceae y Pottiaceae; en segundo lugar se hallan los epífitos (corteza, hojas), con especies de las familias Macromitriaceae, Sematophyllaceae y Pilotrichaceae; finalmente están aquellos que crecen sobre materia orgánica (troncos y ramas en descomposición y hojarasca), con especies de las familias Dicranaceae y Pilotrichaceae (Aguirre, 2008). Como consecuencia del recambio de especies que se presenta entre comunidades de áreas geográficamente distanciadas, los Montes de María comparten solamente seis especies con el valle del Patía (García et al., 2015): Philonotis uncinata (sobre suelo), Erpodium coronatum, Fabronia ciliaris (materia orgánica en descomposición), Neckeropsis undulata (corteza), Hyophila involuta (suelo) y Thuidium tomentosum (roca). 
Los musgos del valle del Patía forman parte de 10 elementos biogeográficos (Anexo 1): 1) Neotropical andino, con 2 especies restringidas a los Andes, 2) Neotropical extraandino, incluye 35 especies con distribución en el Neotrópico, tanto en los Andes como en otras áreas, 3) Pantropical, con 8 especies presentes tanto en el Neotrópico como en el Paleotrópico, 4) Afroamericano, con 5 elementos distribuidos en América y en África, 5) Neotropical-holártico, constituido por 3 especies presentes tanto en el Neotrópico como en la región holártica. 6) Neotropical-austral Antártico, representado por 3 especies comunes a la región tropical americana y a la zona austral, 7) Pantropical-templado, con 3 especies presentes en todo el trópico y en las zonas templadas de ambos hemisferios, 8) Pantropical-holártico, con 1 especie distribuida en el trópico y en la región boreal, 9) Pantropical-austral Antártico, con 2 especies propias del trópico y de la zona templada austral, 10) Cosmopolita, representado por 11 especies que se distribuyen en casi la totalidad del globo.

El elemento dominante es el neotropical extraandino, el cual incluye especies que se distribuyen en las Antillas, México, Centroamérica, el sur de Venezuela, cuenca Amazónica hasta las regiones costeras de Venezuela y Guayanas; también se extienden hasta Bolivia y ocasionalmente hasta Argentina y Chile; los elementos con menor representatividad son el pantropical-austral Antártico y el pantropical-holártico. La presencia de elementos biogeográficos en un área determinada, y específicamente en el valle del Patía, se debe, indudablemente, a múltiples factores tales como la posible existencia de remanentes gondwanianos y laurásicos (Delgadillo, 2000), eventos históricos que ligan a las Antillas con Centroamérica, la formación del Istmo de Panamá y de la cordillera centroamericana (Churchill \& Linares, 1995; Delgadillo, 2000), la formación de la cordillera de los Andes (van der Hammen, 1989) y la dispersión moderada.

\section{Conclusiones}

La presencia de 75 especies de musgos en el valle seco del Patía es una muestra de la escasamente conocida riqueza de briófitos en el bosque seco colombiano y de la necesidad de la realización de otras investigaciones tendientes a obtener un catálogo nacional de musgos presentes en este tipo de ecosistemas. El 46.6 \% de las especies pertenecen al elemento biogeográfico neotropical extraandino que incluye a taxones que se hallan tanto en la región andina como en el resto del trópico americano. En el valle del Patía no existe una preferencia notoria de hábitats por parte de los musgos; no obstante se nota una tendencia a establecerse en áreas con vegetación natural, a lo largo de las corrientes de agua y en bosques y matorrales situados especialmente en cañadas. La mayoría de especies $(60 \%)$ se establecen en un solo tipo de sustrato; las restantes (40\%) colonizan 2 o más sustratos. El número de especies de musgos presentes en este tipo de hábitats puede considerarse como un indicador del estado de conservación del ecosistema.

Doce especies corresponden a primeros registros para el departamento del Cauca, cuatro lo son para el departamento de Nariño; una de ellas (Erpodium beccarii) lo es para Colombia.

Son escasos los especímenes de briófitos de áreas secas en los herbarios nacionales que permitan tener un conocimiento preciso sobre la brioflora de estos ecosistemas; es imprescindible que los herbarios y otras instituciones aborden la investigación en estas áreas para complementar el presente estudio y tener información sobre importancia ecológica y aspectos fisiológicos de este grupo de plantas.

\section{Agradecimientos}

Los autores desean expresar sus agradecimientos al Herbario de la Universidad del Cauca (CAUP) por facilitar las instalaciones, equipos y material vegetal que permitieron realizar las consultas y determinaciones necesarias. Un agradecimiento especial a dos revisores anónimos y al editor quienes hicieron sugerencias y recomendaciones para mejorar el presente documento. También un agradecimiento a todas aquellas personas que de una $u$ otra manera aportaron para la realización de esta investigación. 


\section{Referencias}

Acebey, A., Gradstein, S. R. \& Krömer, T. (2003). Species richness and habitat diversification of bryophytes in submontane rain forest and fallows of Bolivia. Journal of Tropical Ecology, 19, 9-18.

Aguirre, J. (2008). Diversidad y riqueza de los musgos en la región natural andina o sistema cordillerano. En Rangel, J. O. (Ed.). Colombia Diversidad Biótica VI: riqueza y diversidad de musgos y líquenes de Colombia. Pp. 19-54. Bogotá: Instituto de Ciencias Naturales.

Aguirre, J. \& Avendaño, K. (2008). Musgos en la región Caribe. En Rangel J. O. (Ed.). Colombia Diversidad Biótica VI: riqueza y diversidad de musgos y líquenes de Colombia. Pp 55-59. Bogotá: Instituto de Ciencias Naturales.

Churchill, S. P. (1989). Bryologia Novo Granatensis. Estudio de los musgos de Colombia. IV. Catálogo nuevo de los musgos de Colombia. Tropical Bryology, 1, 95-133.

Churchill, S. P. (1991). The floristic composition and elevational distribution of Colombian mosses. The Bryologist, 94, 157-167.

Churchill, S. P. \& Linares C., L. E. (1995). Prodromus Bryologiae Novo-Granatensis. Introducción a los musgos de Colombia. Vol. I y II. Biblioteca José Jerónimo Triana 12, $924 \mathrm{p}$.

Churchill, S. P. (2009). Moss diversity and endemism of the tropical Andes. Annals of the Missouri Botanical Garden, 96, 434-449.

Churchill, S. P. (2016). Bryophyta. En Bernal, R., Gradstein, S. R. \& Celis, M. (Eds.). Catálogo de plantas y líquenes de Colombia. Vol. 1. Pp. 353-442. Bogotá: Instituto de Ciencias Naturales, Universidad Nacional de Colombia.

Clark, T. A. (2012). Bryophyte floristics and ecology in Grand Canyon National Park. Natural Resource (Technical Report NPS/SCPN/NRTR - 2012/602). Fort Collins, Colorado: National Park Service. 88 p.

Corrales, A., Duque, A., Uribe, J. \& Londoño, V. (2010). Abundance and diversity patterns of terrestrial bryophyte species in secondary and planted mon- tane forests in the northern portion of the Central Cordillera of Colombia. The Bryologist, 113(1), 8-21. CRC (Corporacion Autónoma Regional del Cauca). (2004). Caracterización biofísica del Patía. (Informe Técnico). Popayán. 130 p.

Dauphin, L. G. \& Grayum, M. H. (2005). Bryophytes of the Santa Elena peninsula and islas Murciélago, Guanacaste, Costa Rica, with special attention to neotropical dry forest habitats. Lanskesteriana, 5(1), 53-61.

Delgadillo, M. C. (2000). Distribución geográfica y diversidad de los musgos neotropicales. Boletín de la Sociedad Botánica de México, 65, 63-70.

Downing, A. J. \& Selkirk, P. M. (1993). Bryophytes on the calcareous soils of Mungo National Park, an arid área on southern central Australia. Great Basin Naturalist, 53(1), 13-23.

Franks, A. J. \& Bergstrom, D. M. (2000). Corticolous bryophytes in microphyll fern forests of south-east Queensland: distribution on Antarctic beech (Nothofagus moorei). Austral Ecology, 25, 386-393.

García, M. S., Basilio B. H., Herazo, V. F., Mercado, G., J. \& Morales, P., M. (2015). Diversidad de briófitos en los Montes de María, Colosó (Sucre, Colombia). Colombia Forestal, 19(1), 41-52.

García, M. S. \& Mercado G., J. D. (2017). Diversidad de briófitos en fragmentos de bosque seco tropical, Montes de María, Sucre, Colombia. Revista Mexicana de Biodiversidad, 88, 824-831.

Griffin III, D. (1990). Floristic of the South American paramo moss flora. Tropical Bryology, 2, 127-132.

Holdridge, L. R. (2010). Ecología basada en zonas de vida. Agroamérica: San José, Costa Rica: Colección Libros y Materiales Educativos. Quinta reimpresión. 216 p.

Holz, I. \& Gradstein, S. R. (2005). Cryptogamic epiphytes in primary and recovering upper montane oak forests of Costa Rica-species richness, community composition and ecology. Plant Ecology, 178, 89-109.

IGAC (Instituto Geográfico Agustín Codazzi). (1992). Cauca, características geográficas. Santafé de Bogotá. 159 p. 
Ignatova, E. A., Kuznetsova, O. I., Milyutina, I. A., Fedosov, V. E. \& Ignatov, M. S. (2017). The genus Fabronia (Fabroniaceae, Bryophyta) in Russia. Arctoa, 26, 11-34. Magill. R. E. (1976). Mosses of Big Bend National Park, Texas. The Bryologist, 79, 269-295.

Makinde, A. M. \& Odu, E. A. (1994). Phenological studies of selected savanna mosses of south-western Nigeria. Experientia, 50, 616-619.

Oyesiku, O. O. (2016). Phenology of three mosses from Upper Ogun, a savanna area of Southwestern Nigeria. Ife Journal of Science, 18(1), 285-292

Pelayo, H. (2010). Contribución al conocimiento de los musgos de la planicie central del Desierto Chihuahuense, Chihuahua, México. En VII Simposio Internacional sobre la Flora Silvestre en Zonas Áridas. Pp. 808-815.

Pinzón, M. \& Linares, E. (2001). Catálogo comentado de los líquenes y briófitos de la región subxerofítica de La Herrera (Mosquera, Cundinamarca). Caldasia, 23(1), 237-246.

Pócs, T. (1982). Tropical forest bryophytes. En Smith, A. J. E. (Ed). Bryophyte Ecology. Pp. 59-104. Londres: Chapman \& Hall.

Proctor, C. F., Oliver, M. J., Wood, A. J., Alpert, P., Stark, L. R., Cleavitt, N. L. \& Mishler, B. D. (2007). Desiccation-tolerance in bryophytes: a review. The Bryologist, 110(4), 595-621.

Ramírez P., B. R. \& Churchill, S. P. (2002). Las briófitas del departamento de Nariño, Colombia: I. Musgos. Tropical Bryology, 21, 23-46.

Ramírez P., B. R. (2013). Riqueza y distribución de musgos en el departamento del Cauca, Colombia. Boletín científico Centro de Museos Museo de Historia Natural, 17(2), 17-57.
Ramírez-Padilla, B. R., Macias-Pinto, D. J. \& Varona-Balcázar, G. (2015). Lista comentada de plantas vasculares del valle seco del río Patía, suroccidente de Colombia. Biota Colombiana, 16(2), 1-50.

Sagar, T. \& Wilson, P. (2009). Niches of common bryophytes in a semi-arid landscape. The Bryologist, 112(1), 30-41.

Sharp, A. J., Crum, H. A. \& Eckel, P. M. (1994). The moss flora of Mexico. Memoirs of The New York Botanical Garden, 69, 1-1113.

Stark, L. R. (2002). Phenology and its repercussions on the reproductive ecology of mosses. The Bryologist, 105, 204-218.

Tullus, T., Tullus, A., Roosaluste, E. \& Tullus, H. (2012). Bryophyte vegetation in young deciduous forest plantations. Baltic Forestry, 18(2), 205-213.

Uribe, J. \& Gradstein, S. R. (1999). Estado del conocimiento de la flora de hepáticas de Colombia. Revista de la Academia Colombiana de Ciencias, 23(87), 315-318.

Van der Hammen, T. (1989). History of the montane forest of the northern Andes. Plant systematics and evolution, 162, 109-114.

Vilas B., S. \& Passos B., C. J. (1998). Briófitas de uma área de Cerrado no município de Alagoinhas, Bahia, Brasil. Tropical Bryology, 15, 101-119.

Weibull, H. (2001). Influence of tree species on the epilithic bryoflora in deciduous forests of Sweden. Journal of Bryology, 23, 55-66.

Weibull, H. \& Rydin, H. (2005). Bryophyte species richness on boulders: effects of area, habitat diversity and covering tree species. Biological Conservation, 122, 71-9. 
Anexo 1. Familias, géneros y especies de musgos presentes en el valle seco del Patía. Disponible en línea: http:/ / revistas.humboldt.org.co/index.php/biota/rt/suppFiles/637/0

\section{Bernardo R. Ramírez-Padilla}

Universidad del Cauca,

Facultad de Ciencias Naturales, Exactas y de la Educación,

Departamento de Biología

Popayán, Colombia

branly@unicauca.edu.co

https://orcid.org/0000-0001-7798-2303

\section{Verónica Solarte-Téllez}

Universidad del Cauca,

Facultad de Ciencias Naturales, Exactas y de la Educación,

Departamento de Biología

Popayán, Colombia

veronicasolarte@unicauca.edu.co

https://orcid.org/0000-0002-2720-3196

\section{Musgos del valle seco del Patía, suroccidente de Colombia: riqueza, ecología y biogeografía}

\section{Paula A. Ramírez-Burbano}

Universidad del Cauca,

Facultad de Ciencias Naturales, Exactas y de la Educación,

Departamento de Biología

Citación del artículo: Ramírez-Padilla, B. R., Solarte-Téllez, V. \& Ramírez-Burbano, P. A. (2018). Musgos del valle seco del Patía, suroccidente de Colombia: riqueza, ecología y biogeografía. Biota Colombiana, 19(2), 2-11. DOI: 10.21068/ c2018.v19n02a01.

Popayán, Colombia

paularb@unicauca.edu.co

Recibido: 13 de abril de 2018

https://orcid.org/0000-0002-2425-7084 